of a pair for heat only. The value of the lamp for purposes of therapeutic irradiation is doubtful, but it will at least give a sun bath qualitatively, if not quantitatively, similar to sun's rays. In this respect it may perhaps be safer than many of the lamps on the market said to provide the more valuable elements of sunshine and a high proportion of ultra-violet rays, the action of which is ill-understood and frequently somewhat startling on the skin and other parts of the anatomy.

These tungsten filament lamps have been suitably adapted for either hospital or private use by Messrs. Theodore Hamblin, Ltd., London, and should be seen in action under average winter weather conditions to be appreciated. The uses which they may subserve, and the list has by no means been exhausted above, may readily be demonstrated. No doubt Messrs. Theodore Hamblin would make suitable modifications to meet individual suggestions. The disadvantages of a somewhat high recurring expenditure should tend to disappear with an increasing demand and improved methods of manufacture.

\title{
FOGGING FOR THE FOCAL INTERVAL OF STURM
}

BY

\section{Norman GLegG}

WIN DSOR

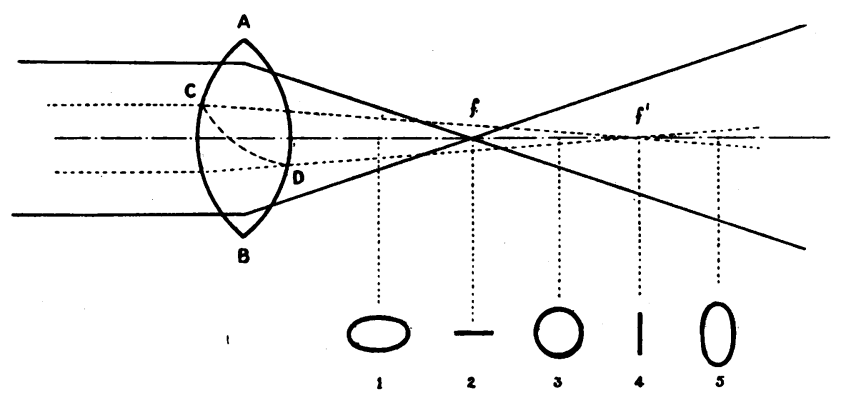

The Focal Interval.

THE following new selective designs in ophthalmic charts provide in a compact and inexpensive form the essentials for analysing refractive errors without a mydriatic. The findings can be suitably modified for the prescription. 
The object of the charts is to facilitate the detection of the axis and amount of astigmatism, and the recording of the visual acuity when corrected for errors of refraction.

The secret of success with all such charts is adequate fogging which similates the use of mydriatics by relaxing the accommodation.

The correct strength of the fogging plus lens is found by retinoscopy, after a preliminary examination of the media and fundi to exclude morbid causes of defective vision, and is the highest plus reading found without reduction for error of one metre working distance. It should at least fog the $6 / 12$ line of type.

With this plus lens in the trial frame of one eye and the other eye occluded, the patient is directed to look at the following charts.

The "Linear" Test Type Chart.

This chart enables the axis and amount of astigmatism to be judged by the relative intensity of blackness of the main axes $(90,180,45,135)$.

\section{The “ANChOR" Chart.}

This chart has the additional refinement of its barbs for 30 , $60,120,150$ axes. Consistent answers to these suggest the findings. With this information
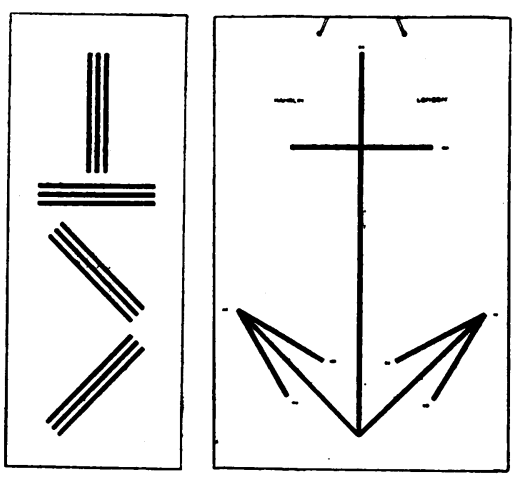
the exact axis and amount of astigmatism can readily be ascertained by reference to:

\section{The "Dial" Chart.}

In this chart the central "V," which rotates, defines to a nicety the exact axis when its limbs are equally blurred. This plus axis is recorded by the indicator at the side of the chart. The amount of the astigmatism is estimated by defogging with minus cylinders in the axis shown by the indicator at the foot of the chart. The linear foci of astigmatism having thus been brought 
to a uniform intensity; a point focus is obtained which can be focussed by minus spheres if necessary till the best visual acuity is obtained as registered by :

The "Spotline" Test Type Chart.

An applied selective value is given to this chart by a simple rearrangement of the usual lines of test types, thus locating for easy reference the desired line and obviating the tiresome habit of the patient's reiteration.

The novelty here is that the most obvious letter $(6 / 60)$ is used as the indicator by being placed between the $6 / 9$ and $6 / 6$ lines which are thus as easily referred to as the $6 / 18$ line
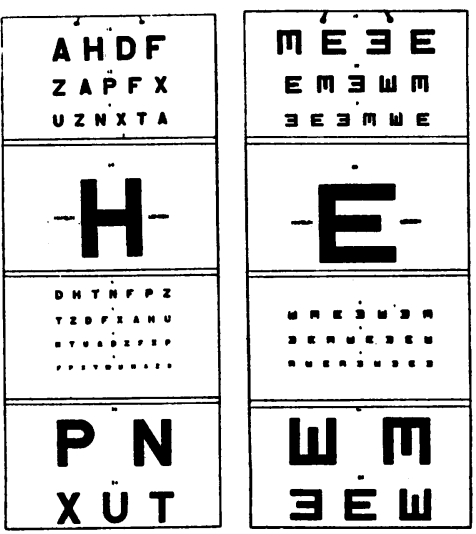
at the top.

While ordinary English, and preferably non-serif, letters may be used, a universally useful character is given to the chart by employing the " $E$ " type, which is equally intelligible to the child, the foreigner or the illiterate, who has only to indicate the direction of the prongs of the "E." Additional advantages of the " $E$ " type are its suitability for both direct or reflected use, also the type can be varied by altering the position of the chart, which can be folded for portability and the Linear type can be printed on its reverse for convenience.

The test type charts must be placed at six metres. The astigmatic charts are more sensitive if displayed at closer range up to even three yards distance.

After many years experience of this method I am sufficiently convinced of its accuracy to desire to encourage its use.

These designs have been produced to my specification by Messrs. Theodore Hamblin, Ltd., of 15, Wigmore Street, London, to whom I am indebted for the line drawings of the various charts which illustrate this article. 